\title{
The development of in situ fracture toughness evaluation techniques in hydrogen environment
}

\author{
John Jy-An Wang*, Fei Ren, Tin Tan, Ken Liu \\ Materials Science and Technology Division, Oak Ridge National Laboratory, Oak Ridge, TN 37831, USA \\ *Corresponding author: Tel: (1)-865-574-2274; email: wangja@ornl.gov.
}

\begin{abstract}
Fracture toughness and fatigue properties of pipeline steels play a critical role in developing advanced high-pressure hydrogen infrastructure for alternative fuel pipelines program. The reliability of structure components, particularly resistance to damage and failure in the intended service environment, is highly dependent on the selected materials. An effective surveillance program is also necessary to monitor the material degradation during the course of service. Steels have been proven to be desirable for hydrogen infrastructure. However, hydrogen embrittlement is an important factor that limits steel performance under high-pressure hydrogen conditions. Furthermore, many conventional fracture testing techniques are difficult to be realized under the presence of hydrogen, in addition to the inherent specimen size effect. Thus it is desired to develop novel in situ fracture toughness evaluation techniques to study the fracture behavior of structural materials in hydrogen environments. In this study, a torsional fixture was developed to utilize Spiral Notch Torsion Test (SNTT) methodology. A fatigue pre-crack procedure of SNTT approach was also developed and demonstrated for weldment. The in situ testing results indicated that the exposure to $\mathrm{H}_{2}$ significantly reduces the fracture toughness of 4340 high strength steels by up to 50 percent. Moreover, in-air simulated heat-affected zone specimen by Gleeble demonstrated a significant fracture toughness reduction in samples which illustrated the effect of welding on the fracture toughness.
\end{abstract}

\section{Keywords -}

Pipeline steel; Welding; Hydrogen; Embrittlement; Fracture toughness, In situ testing; Torsion test; Fatigue test.

\section{INTRODUCTION}

\subsection{Background on Fracture Toughness Evaluation}

The Mode I (tensile opening mode) stress-intensity factor at the onset of rapid crack propagation under plane-strain conditions is defined as fracture toughness, $\mathrm{K}_{\mathrm{IC}}$, a controlling reference parameter used in design to avoid catastrophic brittle fracture. American Society for Testing and Materials (ASTM) standard test methods, Standard Test Method for Plane-Strain Fracture Toughness of Metallic Materials (E399) [1], Standard Test Method for Measurement of Fracture Toughness (E1820), and Standard Test Method for Determining Threshold Stress Intensity Factor for Environment-Assisted Cracking of Metallic Materials (E1681-03), are widely used to determine fracture toughness of metallic materials, using compact tension and compact disk tension specimens, and wedge opening load specimen, having thickness and volume sufficient to ensure the plane-strain condition at the crack front. However, meeting the specimen size requirements is difficult and impractical because structure systems materials to be investigated may be geometrically unsuitable and/or have insufficient volume for making the standard specimen. Therefore, use of small specimens for $\mathrm{K}_{\mathrm{IC}}$ measurement is essential for application to pressure vessel safety surveillance.

Despite the international efforts on the development of small specimen testing techniques, no methods currently exist for direct measurement of $\mathrm{K}_{\mathrm{IC}}$ for small specimens without a concern for size effect. A new method, designated as Spiral Notch Torsion Test (SNTT), is developed recently by the authors to measure the intrinsic fracture toughness $\left(\mathrm{K}_{\mathrm{IC}}\right)$ of structural materials [2-6]. The SNTT overcomes many of the limitations inherent in traditional techniques and makes it possible to standardize fracture toughness testing. The SNTT system operates by applying pure torsion to uniform cylindrical specimens with a notch line that spirals around the specimen at a $45^{\circ}$ pitch. The $\mathrm{K}_{\mathrm{IC}}$ values are obtained with the aid of a three-dimensional finite-element computer code. 
In the old days, welds were almost always blamed for any structure-joint failure [7]. But researchers soon identified another suspicious region, heat-affected zone (HAZ), between the steel and the weld [8-9]. And they realized it was this vulnerable HAZ that had been causing much of the trouble. The detailed microstructure and the associated complex fracture morphology of weldment are shown in Fig. 1. The high hardness contours shown in Fig. 1a are associated with HAZ materials. The weld microstructure consisted of 3 regions; i.e., weld metal, HAZ, and base metal. The HAZ can be further divided into several sub-regions with obviously different microstructures. The HAZ microstructure of a singe-pass weld on martensitic steel can be defined as follow:

HAZ-1: subcritical region, where microstructure similar to that of the base metal, HAZ-2: constitute the very fine microstructure, HAZ-3: the grains are finer than that of base metal, but larger than in HAZ-2,

HAZ-4: coarse grain region located next to the fusion line, the highest hardness resided for
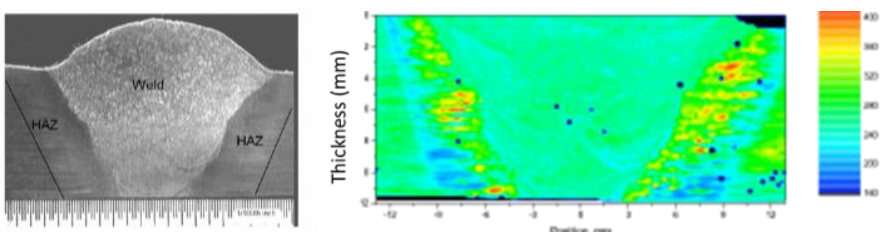

(a)
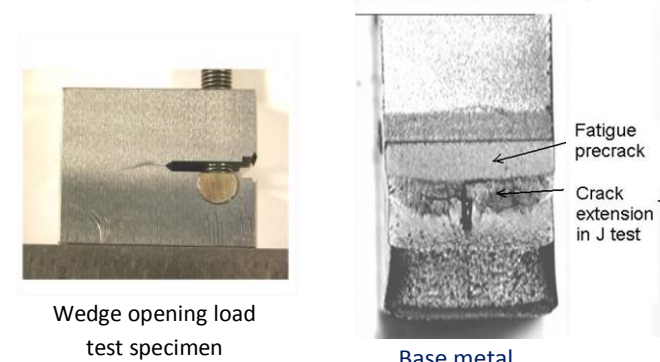

Base metal

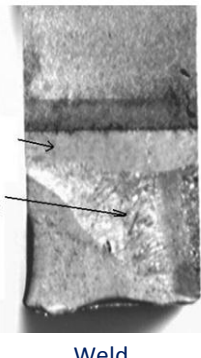

(b)

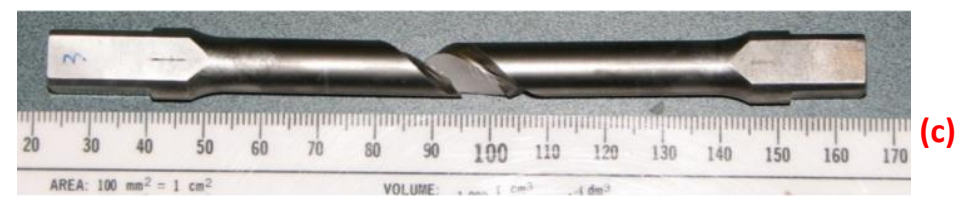

Figure 1: (a) Distribution of micro-hardness in a weld cross section, (b) fracture surface appearances of high strength pipeline steel in fracture toughness testing, significant distortion of crack growth was observed from weld CT specimen, (c) Fractured SNTT weld sample shows smooth and uniform fracture surface contour.

ferrite steel.

Complications in HAZ testing and enormous data scatter have discouraged researchers from addressing the subject of HAZ fracture toughness. [8-12] For instance, to generate a credible HAZ fracture toughness data, it will require that the tip of the fatigue-precrack located at the coarse-grain region of the HAZ specimens. However, in general, the fatigue crack is advanced toward the soft part of metal; therefore, avoid the brittle coarse-grain region of HAZ. The lack of details of material properties for weld-HAZ, in addition to no consensus standards for HAZs toughness evaluation, has cast a great uncertainty regarding the integrity assessment of the weldment. Furthermore, pressure vessel steel weld-HAZ has potential for reheat cracking in the grain growth zone [13], and this reheat cracking is not only confined to the transformable steels but also in Ni-based alloys and austenitic stainless steels. Also, cold cracking (or hydrogen induced cracking) [14] and lamellar tearing are typically appeared in the HAZ. An integrated technology to resolve weld-HAZ fracture behavior and toughness evaluation is critically needed. Accurate determination of the HAZ interfacial fracture toughness/cohesion bond will be immensely helpful to understand weld material aging degradation or recovery occurring in the course of service. Knowing the chronological changes in the HAZ fracture toughness will lead to effective control and improvements in HAZ material properties through proper processing and post-weld heat treatment. Because the direction of crack growth is predictable and controllable during a SNTT fatigue pre-crack procedure, the tip of crack front then has opportunity resides at point of interest, thus, $\mathrm{K}_{\mathrm{IC}}$ values can be reliably determined for functionally gradient materials, weldment, and polymeric composites, using the SNTT method. [15-16] Therefore, the SNTT method is a powerful technique for investigating the interfacial material properties at HAZ and functionally gradient materials.

Durability and reliability of structural materials significantly affects structural integrity and safety of the hydrogen infrastructures. For efficiently operation, many topics must be explored to ensure the structural safety, in particular, the basic understanding of damage and failure mechanisms, effects and interpretation of the service or stochastic loadings, multiple stress states, environmental effects, size effects, and thickness effects. Reliable evaluation of the durability is highly desired to provide a crucial understanding of various fracture mechanisms so that improved structural designs and optimized materials selection can be continuously achieved. The proposed new testing protocol based on SNTT methodology was initiated, shown in Figure 2, to meet this need. Fig. 2a showed that the principle 
tensile stress (opening mode) is perpendicular to the $45^{\circ}$ spiral groove line, and crack propagation is toward and perpendicular to the specimen central axis. Fig. 2c illustrated SNTT specimen minimization; Fig. 2 d illustrated the SNTT is direct transformation of CT specimen; and Fig. e and Fig. f are the extension of SNTT in interface fracture toughness investigation of alumina oxide coated SNTT sample.

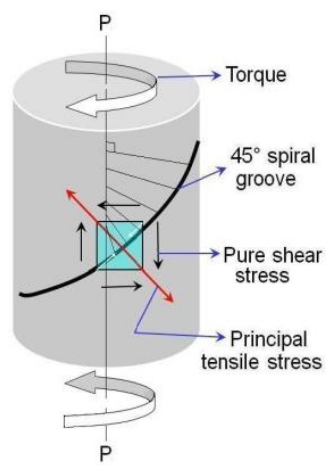

a) schematic of the theory

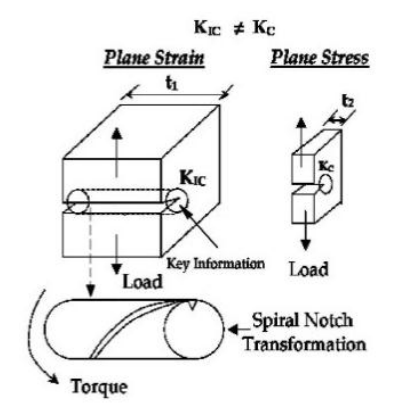

d) CT and SNTT specimens

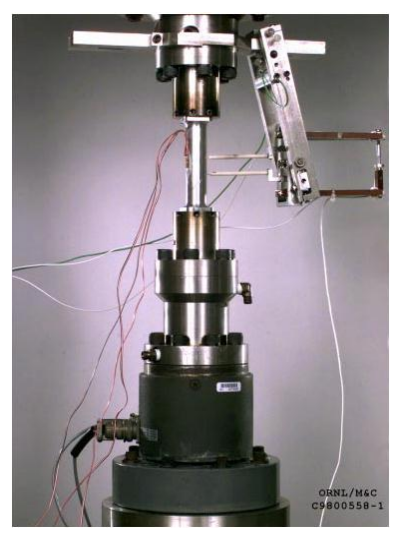

b) SNTT fracture test setup

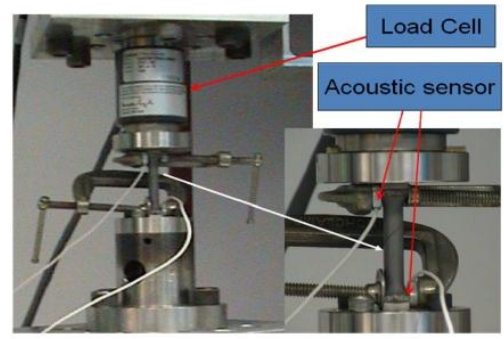

e) SNTT coating test setup

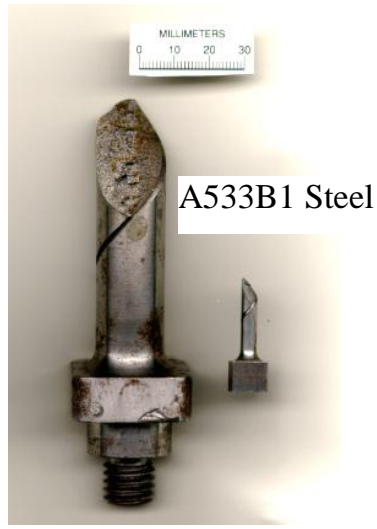

c) halves of tested A302B specimens

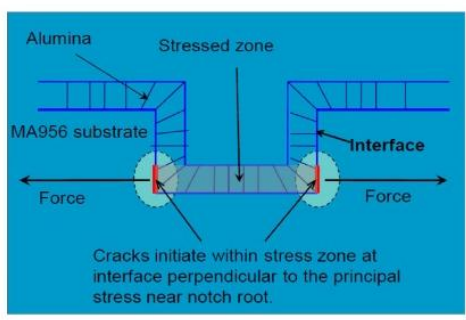

f) U-grooved spiral coating test

Figure 2: The developed SNTT technology

Polymeric composite has been used in structural components; due to its inhomogeneous material nature, its system durability and reliability deserve special attention. The weakest link theory approach of the spiral notch concept was successfully developed and applied earlier in the interface fracture toughness evaluation for the thin film coating SNTT samples [15] as well as in the interface cohesion bond study for polymeric composite SNTT samples [16], where the interfaces at the material mismatch region (or dissimilar materials) provide the stress riser sites (that can be resembled to an internal notch or flaw) and under cyclic fatigue loading, these interfaces in term become the crack initiation sites. In another recent effort [17], we also successfully demonstrate the similar concept with a SNTT epoxy sample, where the spiral groove was filled with the fiberglass and then cured with epoxy to bond to SNTT epoxy sample. The formed SNTT sample is shown in Fig. 3, where the strip (non-clear darker zone) is the reinforced fiberglass region. The fatigue pre-crack procedure was applied to this SNTT sample (w/o machined notch) to induce the fatigue crack growth. The fatigued sample in Fig. 3 shows that fatigues crack initiation sites are all originated at the interface between SNTT epoxy matrix and the boundary of reinforced fiber strip. A similar interface crack was also observed for SNTT composite sample with epoxy resin infused into spiral groove under fatigue cycle. The approach of utilizing the internal weakest interfaces or material mismatch sites to trigger the crack initiation or the follow-on crack growth demonstrated above will be applied to HAZ SNTT specimens to induce fatigue pre-crack, in addition to spiral groove machined to the SNTT specimens near weld-HAZ region for
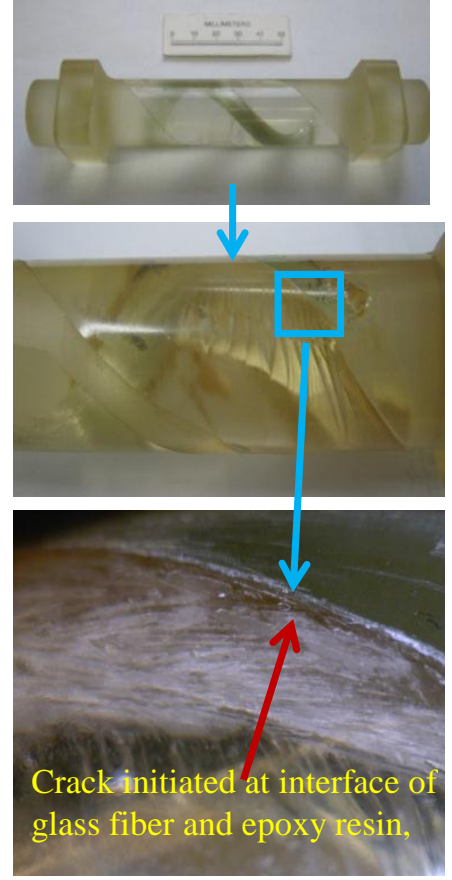

Fig. 3 Epoxy SNTT Sample with Glass Fiber Embedded. comparison purpose. 
Piping systems used in the hydrogen infrastructure cannot be constructed without curve-shaped components, such as elbows, U-bends and T-joints. While these components are effective in providing system flexibility to mitigate flexural loading (mode I loading), they also create torsional load (shear load known as mode III loading, induced by the out-of-plane loading) to the piping system. Fracture behavior under mixed mode loading (modes I and III) is not well known partially due to the experimental difficulties with the test method using a CT specimen. A testing protocol uses a modified CT specimen and complicated loading grip system to pursue Mode I + Mode III fracture toughness evaluation. The test result for ductile metals shows that at the critical angle of the Mode I + Mode III loading the associated mixed-mode fracture toughness can drop to 50\% of mode I only fracture toughness. [18]. The SNTT method has a significant advantage over the conventional methods to accomplish mixed mode fracture toughness testing by either using different pitch angle of the spiral groove or applying various combinations of loads in tension and torsion to the standard specimen.

Because of the service environment and structural design, the hydrogen infrastructure material is often subjected to combined flexural tensile and shear/twisting loads and suffers potential mixed-mode damages that can eventually lead to fracture, particularly after the material has experienced long-term aging in service. For conventional technologies, the highly desired realistic simulation and reliable evaluation of structural material working conditions and mixed-mode factures constitute a significant challenge. The common use of uniaxial tensile, compressive, and fatigue specimens cannot simulate the complicated multi-axial stress conditions, and approximations in common practice mostly result in either risky or over conservative evaluations. Other conventional approaches, such as the use of the disk compact tension specimen and the compact tension (CT) specimen which is accepted as an ASTM standard, require considerable specimen thickness and in-plane dimensions to ensure plane strain conditions at the crack front for ductile materials. For structural materials that are thin and unrealistic for using thick and big specimen to simulate, not meeting the ASTM minimum specimen size inevitably leads to unreliable results. Further, the CT specimen also has an inherent problem of indeterminately non-uniform load distribution along its width due to stress anomaly near the two free surfaces at the ends of the crack front. The consequence of this is non-uniform crack front, such as in a thumbnail or zigzag crack front form; especially for typical weld materials which normally show significant distorted crack front from the fracture mechanics testing.

The SNTT technology overcomes these difficulties with the following technical and economical superiorities to conventional technologies:

1. It can efficiently generate multiple modes of stress (e.g., flexural normal stress and torsional shear stress) simultaneously to provide realistic simulation of hydrogen infrastructure system (HYS) working conditions and reliable evaluation of durability of the HYS materials and system designs;

2. It eliminates the indeterminately non-uniform load distribution along the crack simulation front, the inherent problem of the ASTM standard CT and other similar technologies.

3. It does not require a large specimen size or thickness to ensure accuracy of the results, and can be adapted for evaluating tubing structure.

4. Its permissible small specimen size offers great economical advantage by avoiding expensive large specimen preparation required for the ASTM standard CT and other similar technologies.

5. Its flexible specimen sizes allow cost-efficient simulation of various HYS material service conditions without entailing large, expensive environmental chambers and control systems.

6. Besides durability against mixed-mode fracture, it can be further developed to investigate the end-effects of material joining under different loading conditions.

7. It also has great potential to simulate and evaluate multi-axial fatigue and other complicated damage mechanisms of HYS materials, by adjusting the axial and torsional loads, in addition to varying the pitch angles of the spiral notch.

8. It has proven capability to test coating adherence, which can be used for evaluating the bonding strength of coating to structural materials and other surface protection.

\subsection{Hydrogen Delivery System}

The hydrogen energy transport and delivery infrastructure will require extensive use of steels and other cost-effective structural and functional materials under high-pressure gaseous hydrogen $\left(\mathrm{H}_{2}\right)$ exposure. For example, high 
pressure (up to $3000 \mathrm{psi}$ ) hydrogen pipelines are presently considered to be one of the most cost-effective and energy-efficient means to transport very large amounts of hydrogen to much of the market as is done currently for natural gas [19]. Under high hydrogen pressures, there are concerns about hydrogen embrittlement (HE) of steel pipelines and its potential catastrophic consequences [20]. Concerns regarding hydrogen embrittlement are not limited to steel pipelines; according to a DOE Basic Energy Science Office report [21], hydrogen embrittlement needs to be addressed for a variety of hydrogen storage and delivery system parts made of metallic materials that are exposed to hydrogen.

As in the case of natural gas and other energy carrier transmission pipelines, welding will be used to construct steel pipelines for high-pressure hydrogen delivery. Welding will be also widely used in fabrication of other system components for hydrogen production, storage, and delivery. However, welds in pipeline steels and other engineering materials are often the most susceptible regions to hydrogen embrittlement due to the formation of unfavorable microstructures and high tensile residual stresses. Indeed, recent studies [22] on pipeline steels have shown that the weld region exhibits delayed cracking (signature of hydrogen embrittlement) when exposed to high-pressure hydrogen gas. Furthermore, the weld region typically has substantially lower resistance to hydrogen crack initiation and higher crack growth rates, when compared to the baseline pipeline steel. In this regard, the weld region can be the weakest link for the structural integrity and safety of hydrogen pipelines and hydrogen delivery infrastructure. A systematic approach to deal with weld property degradation under high-pressure hydrogen gas is critical to ensure the safe, cost-effective operation and long-term reliability of the hydrogen delivery infrastructure. [23]

This paper presents the progress to date in the following four areas: 1) application of Spiral Notch Torsion Test (SNTT) on the fracture study of pipeline steels and welds; 2) development of fatigue pre-crack procedure for ductile metals; 3) development of in situ hydrogen testing apparatus for SNTT tests; and 4) mechanical evaluation of base and welded materials in hydrogen environments.

\section{SPIRAL NOTCH TORSION TEST}

Fig. 4 illustrates the stress distribution state along the $45^{\circ}$ spiral line on a cylindrical surface subject to pure torsional load, and its potential crack propagation contour of a SNTT sample. When a notch is introduced along the spiral line, the region near the notch is then subject to a tri-axial tensile stress state [2]. Thus, the effective loading modes are Mode I loading (opening mode) when the pitch angle of the notch is $45^{\circ}$, Mode III loading (tearing mode) when the pitch angle is $90^{\circ}$, and mixed mode loading (Mode I+Mode III) at pitch angle between $45^{\circ}$ and $90^{\circ}$.

\subsection{A302B Steel Testing}

The A302B specimen has a uniform gage section of 20.3-mm diameter and 76.2-mm gauge length. An A302B specimen having a spiral V-groove with a depth of 1.9-mm was tested. A fatigue precrack procedure was used to control crack growth with reference to the change in slope of the load-displacement curves. The specimen (Fig. 2c) fractured at $519.7 \mathrm{~N} \cdot \mathrm{m}$ torque at room temperature. Postmortem examination indicates that the final failure mode is brittle fracture. A fatigue precrack protocol for using miniature SNTT samples of A533B steel was also successfully developed. A comparison of fractures obtained from the prototype and miniature specimens is illustrated in

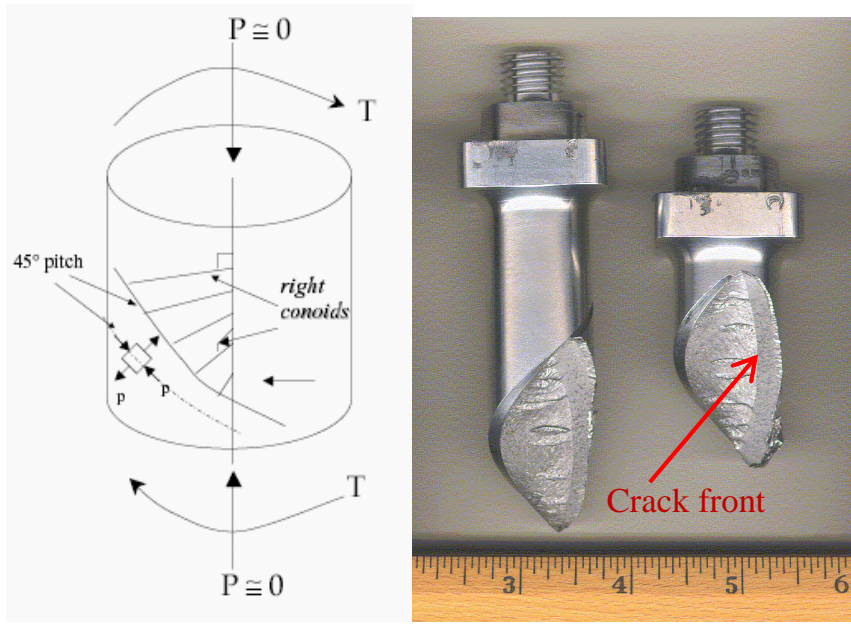

Figure 4: (Left) Pure torsion generates an equibiaxial tension /compression stress field on $\pm 45^{\circ}$ - pitched orthogonal planes along right conoids, independent of specimen diameter; a plane strain condition is maintained on every plane normal to the spiral groove. (Right) Fractured pair of a SNTT sample, made of 7475-T7351 aluminum, shows uniform crack front and crack propagation is perpendicular to the specimen center axis. Fig 2c. 


\subsection{Weld Specimen Testing}

X52 weld SNTT samples were fabricated from a segment of friction stir welded X52 steel pipe. The thickness of the pipe is 0.5 inch and the diameter of SNTT samples was designed as 0.375 inch accordingly. The SNTT specimen axis was parallel to the pipe cylinder axis. One complete loop of spiral groove was machined on the X52 SNTT sample. The fractured X52 baseline SNTT sample is shown in Fig. 5 (a), the detailed fatigue precrack area is shown in Fig. 5(b), and the cross-section of fractured X52 weld SNTT sample is illustrated in Fig.5(c). [24]

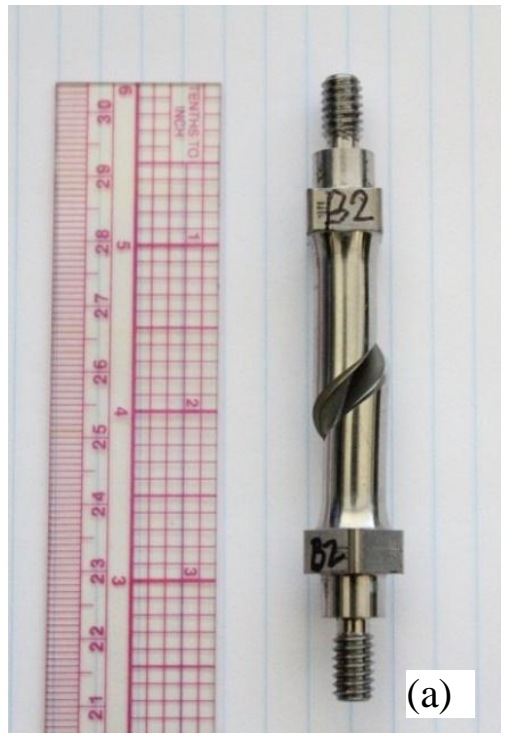

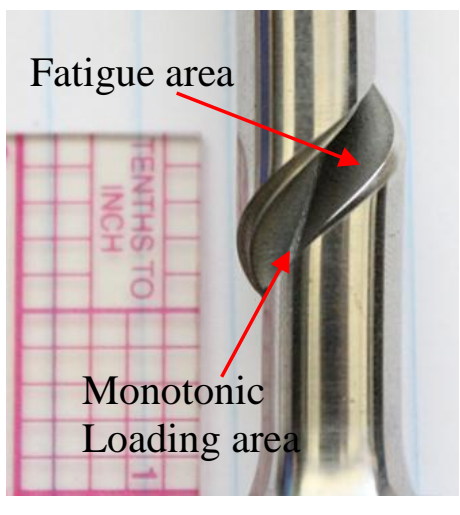

(b)

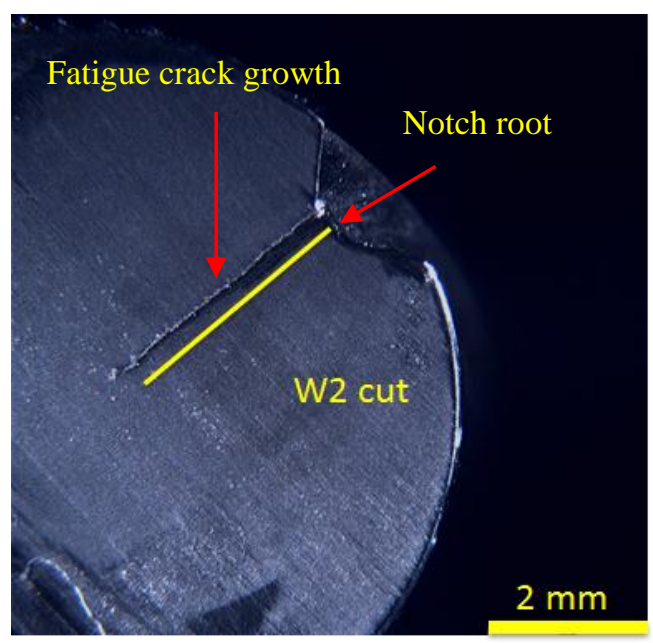

(c)

Figure 5 The failed specimen of X52 baseline sample, (a) entire view; (b) enlarged view of the middle section of tested SNTT sample, (c) the fatigue pre-crack growth profile of X52 weld SNTT sample W2, where the crack growth orientation is toward the central axis of the SNTT sample.

\section{DEVELOPMENT OF SNTT FATIGUE PROTOCOLS}

In addition to single notch front geometry, the crack growth behavior of SNTT protocols has been effectively established using an integrated experimental, numerical and analytical approach. The results indicate that the proposed protocol not only provides significant advance in understanding the compliance evolution of the SNTT specimen, but also can be readily utilized to assist future development of hydrogen infrastructures.

SNTT samples were machined directly from the existing pipelines. For each sample, an entire spiral loop was machined for the SNTT cylinder with a notch-to-diameter ratio of 0.1 . A new set of fixtures was fabricated to accommodate the X52 SNTT samples. Threads were introduced into both ends of the SNTT samples (shown in Fig. 5a) to help improve sample

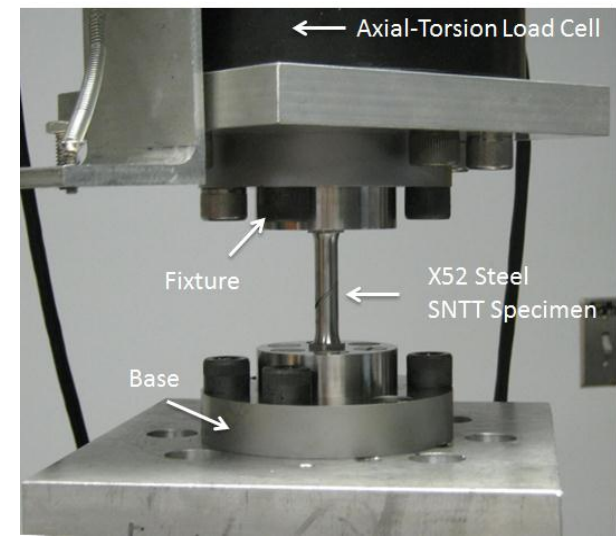

Figure 6. SNTT testing setup for X52 steel specimen. stability and to ensure nil zero axial loading during the cycle-fatigue process. SNTT test set-up of X52 is shown in Fig. 6.

The finite element method (FEM) was used to characterize the crack growth during SNTT fatigue pre-crack testing. FEM models were established to capture stress concentrations around non-coplanar three-dimensional spiral crack front; the typical butterfly stress contour was observed near the crack front shown in Fig. 7. A series of FEM models was

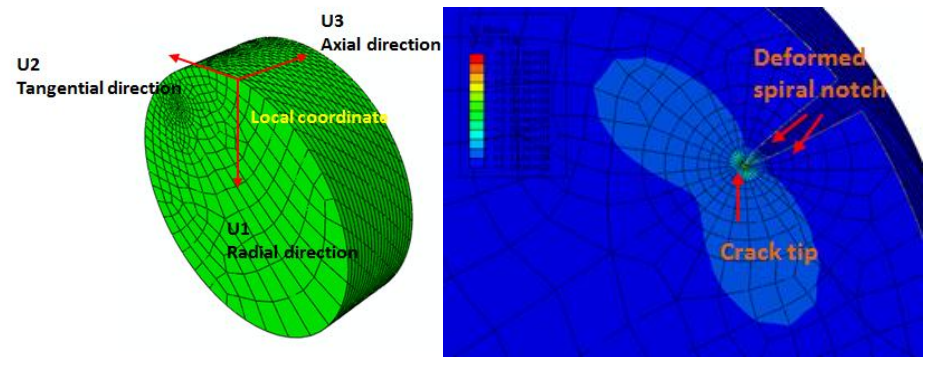

Fig. 7 (Left) FEM model and (right) von Mises stress contours near the crack tip of steel SNTT sample. 
created to cover the crack depth range from 0.1 to 0.45 in the crack depth-to-diameter ratio. For each crack depth, either rotation angle or torque was applied to perform the numerical analysis. The output from these models included the reaction torque, the end rotational angle, and the energy release rate. Beside the tested SNTT cylinder samples, which measured 0.375 inch in diameter, numerical cases were also extended to SNTT samples with a 1-inch diameter. Corresponding torsional load and deformation parameters, including the torque, angle and energy release rates, were also obtained for these models. Evaluation was also performed on aluminum SNTT samples with the above torsional parameter, and the finite element results were included for further analysis.

By summarizing these numerical results, the evolution of the SNTT compliance and the energy release rates were studied carefully with respect to different crack lengths. Two non-dimensional indices, the characteristic compliance and the characteristic energy release rates of SNTT, were developed to quantify the crack growth process of SNTT. Overlapped trends were observed between SNTT samples with different dimensions, as well as between samples made from both steel and aluminum. Analytical models in both broken and unbroken ligament forms were proposed to quantify the crack penetration depth based on these non-dimensional indices.

Experiment measurements were performed at different crack lengths during the cycle fatigue process. Specimens were cut in cross sections, and crack penetration depths were compared with predictions from the compliance function. For the base material X52, a generally good agreement was observed between predictions and the experimental measurement. For the welded X52 materials, good agreements were observed for some specimens. For other weld samples, crack penetration deviation was observed during the cycle fatigue process. The reason for this deviation is probably related to the heterogeneous properties of the welded material, on which further study is needed. In order to validate the analytical models, further analysis was carried out on the rotational angle measurement and finite element model predictions. The net section of the SNTT specimen was modeled with the measured crack depth. By using the torque data from the RVDT measurement, good agreement was observed between the predictions and the measurement.

\subsection{The Evolution of Compliance and Fracture Resistance in SNTT Process}

Systematic studies reveal that an SNTT-type specimen (a rod-type specimen having a helical groove with a 45-degree pitch) can effectively simulate the behavior of a thick compact-tension specimen equal to the total length of the groove line. Careful studies show that the evolutions of compliance and fracture resistance of the SNTT sample during the crack growth process can be unified together irrespective of specimen sizes and material types. In addition to the special features of small volume specimen and ease of testing with the SNTT method, the independence of size effect is in rigorous analytical results for this testing method. The evolution of compliance and fracture resistance in the SNTT process has also been presented with simple governing equations using the ratios of crack lengths over the cylindrical diameter. Based on the measured torques and rotation angles, the penetrated crack depth can be obtained through compliance governing equations. Once crack depths are known, the energy release rates, i.e., the crack driving force, can be obtained from the fracture resistance equation for the SNTT experiments. Therefore, it is possible to control the crack penetration depth in SNTT experiments via monitoring the applied torques and angles, a technique easily adapted by industry.

Figure 8 shows the evolution of crack growth in an SNTT sample with a cylinder diameter of 1 inch, where $a$ is the crack length and $\mathrm{D}$ is the diameter. The ratio of crack length over diameter increases from 0.10 to 0.45 . The length of each model is the same during the crack growth process. Figure 8a shows the compliance evolution of the SNTT sample with respect to different ratios of crack length over diameter. To obtain a more sensitive response of the compliance evolution, a factor was applied to account for the effect of unbroken ligament of the SNTT samples, which is graphed in Figure 8b. It shows that the compliance evolution curves are the same for specimens with different materials or sizes. $\gamma$ is the unit end rotation angle; T is the applied torque; $\mu$ is the shear modulus; and $\mathrm{R}$ is the cylinder radius. The non-dimensional compliance index developed in Fig. 8 can be used to estimate the crack penetration depth of pre-cracked SNTT specimen under the targeted torque and unit rotation angle.

Figure 9a shows the fracture resistance evolution of the SNTT sample with respect to crack length over diameter ratios. In order to obtain a more sensitive response of the compliance evolution, a factor also was applied to account 
for the effect of unbroken ligament of the SNTT samples, which is shown in Figure 9b. It was noted that non-dimensional fracture resistance curves are the same for specimens with different materials or sizes. $G$ is the energy release rate; $\mathrm{T}$ is the applied torque; $\theta$ is the associated rotation angle; Area is the cross section area of the cylinder. The non-dimensional energy release rate index developed in Fig. 9 can be used to estimate the energy release rate of pre-cracked SNTT specimen at the designated crack penetration under the targeted torque and rotation angle.

$$
\begin{aligned}
& \text { Unscaled SNTT Crack Growth Compliance Equation } \\
& \frac{\gamma}{\mathrm{T}} \mu \mathrm{R}^{4}=-0.6541\left(\frac{\mathrm{a}}{\mathrm{D}}\right)^{4}+0.5189\left(\frac{\mathrm{a}}{\mathrm{D}}\right)^{3}+1.7171\left(\frac{\mathrm{a}}{\mathrm{D}}\right)^{2}+0.2045\left(\frac{\mathrm{a}}{\mathrm{D}}\right)+0.6254
\end{aligned}
$$

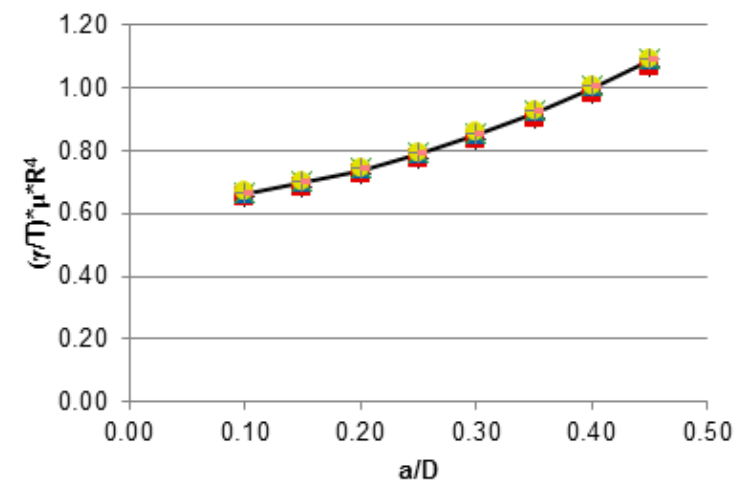

- Steel theta $=0.0015$ (1 inch)

- Steel Torque $=5000 \mathrm{lbf}-$ in ( 1 inch)

$\Delta$ Al theta $=0.002$ ( 1 inch)

Al Torque $=2500 \mathrm{lbf}$-in ( 1 inch)

* Steel Theta $=0.002$ ( 0.375 inch)

- Steel Torque $=300 \mathrm{lbf}-$ in $(0.375 \mathrm{inch})$

+ Al Theta $=0.0025$ (0.375 inch)

- Al Torque=150 lbf-in (0.375 inch)

(a)

\section{Scaled SNTT Crack Growth Compliance Equation}

$$
\frac{\gamma}{T} \mu R^{4}\left(1-\frac{\mathrm{a}}{D}\right)^{4}=3.3445\left(\frac{\mathrm{a}}{D}\right)^{4}-5.2514\left(\frac{\mathrm{a}}{D}\right)^{3}+4.0568\left(\frac{\mathrm{a}}{D}\right)^{2}-2.2298\left(\frac{\mathrm{a}}{D}\right)+0.6226
$$

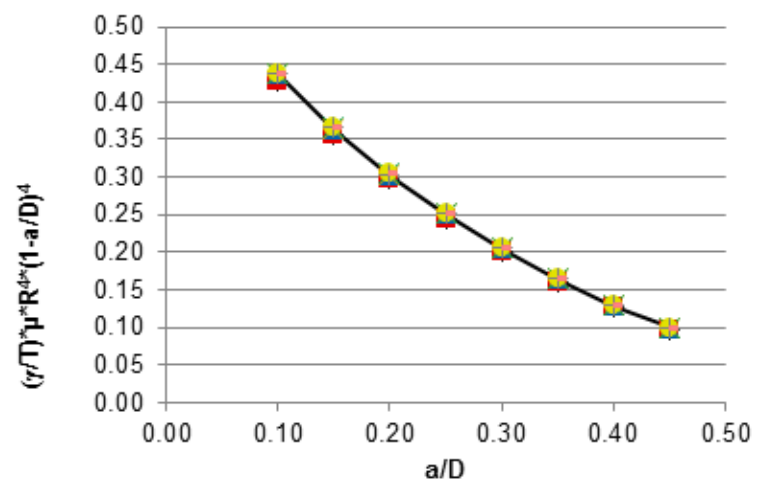

- Steel theta $=0.0015$ ( 1 inch)

- Steel Torque $=5000 \mathrm{lbf}$-in ( 1 inch)

$\Delta$ Al theta $=0.002$ ( 1 inch)

Al Torque $=2500 \mathrm{lbf}$-in ( 1 inch)

* Steel Theta $=0.002$ ( 0.375 inch)

- Steel Torque $=300 \mathrm{lbf}-$ in $(0.375 \mathrm{inch})$

+ Al Theta $=0.0025$ ( 0.375 inch)

- Al Torque $=150 \mathrm{lbf}$-in (0.375 inch)

(b)

Figure 8. (a) The unscaled compliance evolution with the crack growth; (b) The scaled compliance evolution along the crack growth with unbroken ligament factor. 


\section{Unscaled SNTT Energy Release Rates Evolution Equation}

$$
\frac{\mathrm{G}^{*} \mathrm{~A}}{T * \theta}=-95.543\left(\frac{\mathrm{a}}{\mathrm{D}}\right)^{4}+107.14\left(\frac{\mathrm{a}}{\mathrm{D}}\right)^{3}-52.72\left(\frac{\mathrm{a}}{\mathrm{D}}\right)^{2}+16.028\left(\frac{\mathrm{a}}{\mathrm{D}}\right)-0.2489
$$

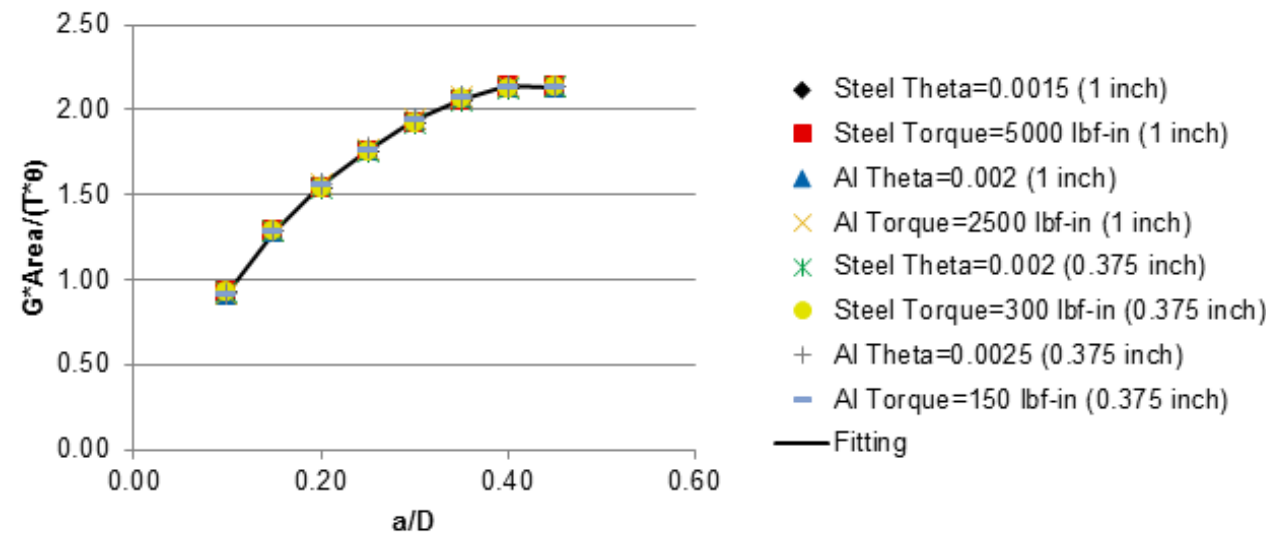

(a)

$$
\begin{aligned}
& \text { Scaled SNTT Energy Release Rates Evolution Equation } \\
& \frac{\mathrm{G}^{*} \mathrm{~A}}{T * \theta *\left(1-\frac{\mathrm{a}}{\mathrm{D}}\right)^{2}}=-154.56\left(\frac{\mathrm{a}}{\mathrm{D}}\right)^{4}+188.95\left(\frac{\mathrm{a}}{\mathrm{D}}\right)^{3}-62.398\left(\frac{\mathrm{a}}{\mathrm{D}}\right)^{2}+20.626\left(\frac{\mathrm{a}}{\mathrm{D}}\right)-0.4716
\end{aligned}
$$

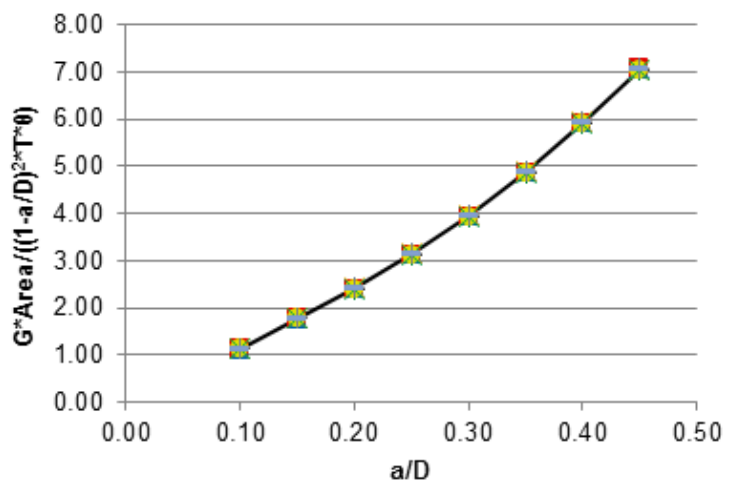

$$
\begin{aligned}
& \text { - Steel Theta }=0.0015 \text { ( } 1 \text { inch }) \\
& \text { - Steel Torque }=5000 \mathrm{lbf} \text {-in }(1 \mathrm{inch}) \\
& \times \text { Al Theta }=0.002(1 \mathrm{inch}) \\
& \text { - Steel Theta }=0.002(0.375 \mathrm{inch}) \\
& \text { * Steel Torque }=300 \mathrm{lbf}-\text { in }(0.375 \mathrm{inch}) \\
& + \text { Al Theta }=0.0025(0.375 \mathrm{inch}) \\
& - \text { Al Torque }=150 \mathrm{lbf}-\text { in }(0.375 \mathrm{inch})
\end{aligned}
$$

(b)

Figure 9. (a) The unscaled energy release rate evolution with the crack growth; (b) The scaled energy release rate evolution along the crack growth with unbroken ligament factor.

\section{SNTT TESTING OF 4340 STEEL IN AIR}

SNTT testing conducted in air is illustrated in Fig. 6, where a biaxial extensometer was used to measure the relative displacement across the spiral notch. To introduce a sharp precrack at the tip of the machined notch, the cyclic fatigue is performed first. The sample with precrack is then loaded by a unidirectional torque to failure.

Three-dimensional finite element modeling was used to assist the calculation of fracture toughness using the measured torque at failure and the precrack length determined from postmortem microscopic examination.

The fractured surface of fatigue pre-crack samples usually consists of a fatigue precrack zone (region "A" in 10a) and 
a region corresponding to the final failure caused by monotonic loading (region " $\mathrm{B}$ " in 10a). Fatigue cracks propagate mainly by transgranular fracture (10b), leading to a fairly smooth precrack surface (10a). A significant amount of cleavage was observed in region " $\mathrm{B}$ " (10c), indicating the brittle feature of the fracture.

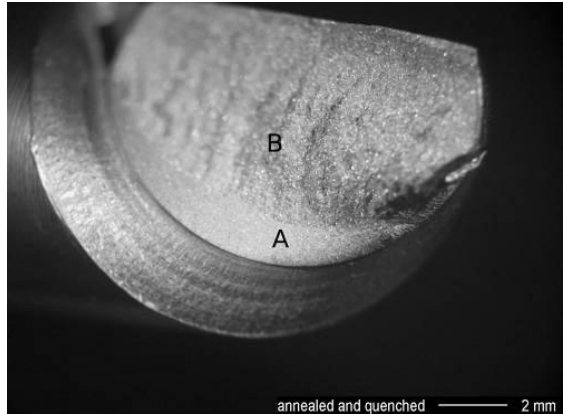

(a)

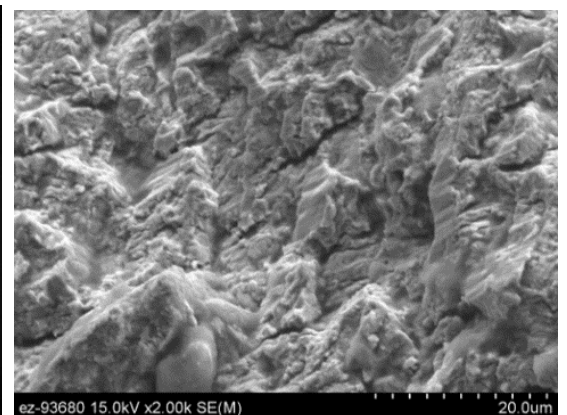

(b)

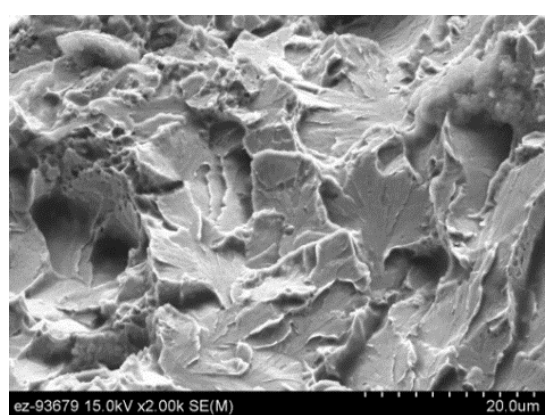

(c)

Figure 10. Micrographs of a quenched sample showing (a) the fracture surface at a low magnification, (b) the fatigue region at a high magnification, and (c) the monotonic fracture region at a high magnification.

A simulated welding heat treatment using a Gleeble system [25] was applied on 4340 steel samples in order to introduce non-uniform microstructures typically encountered in a weld heat affected zone (HAZ). In addition, normalized and oil quenched samples were included for the purpose of comparison. The heat treatment details were given elsewhere [26].

It was observed that fracture toughness values $\left(\mathrm{K}_{\mathrm{IC}}\right)$ of Gleebled samples were reduced by up to $52 \%$ (Table 1), which is consistent with our previous observation of samples without precrack [26]. In addition, the comparison between fatigue precrack and non-fatigue precrack sample results demonstrates that introducing fatigue precracks can significantly reduce the contribution from plastic deformation during crack propagation in the measured fracture toughness value.
Table 1. Comparison of fracture toughness values of 4340 steel SNTT samples subject to different heat treatments as tested in air.

\begin{tabular}{|l|l|l|}
\hline Heat treatment & Precrack $(\mathrm{mm})$ & $\mathrm{K}_{\mathrm{IC}}\left(\mathrm{ksi}-\mathrm{in}^{1 / 2}\right)$ \\
\hline Quench & 1.34 & 51.7 \\
\hline Gleeble & 0.74 & 24.7 \\
\hline Quench & No precrack & 76.5 \\
\hline Gleeble & No precrack & 47.1 \\
\hline
\end{tabular}

\section{DEVELOPMENT OF IN SITU TESTING APPARATUS FOR SNTT TESTING}

The successful application of SNTT in air encouraged the development of in situ apparatus for testing under hydrogen environments. A torsion load frame/sample holder (Fig. 11a) with the capability of applying preload on the sample (Fig. 11b) was designed and fabricated [27], which can be submerged in a hydrogen tank, shown in Fig. 11(c).

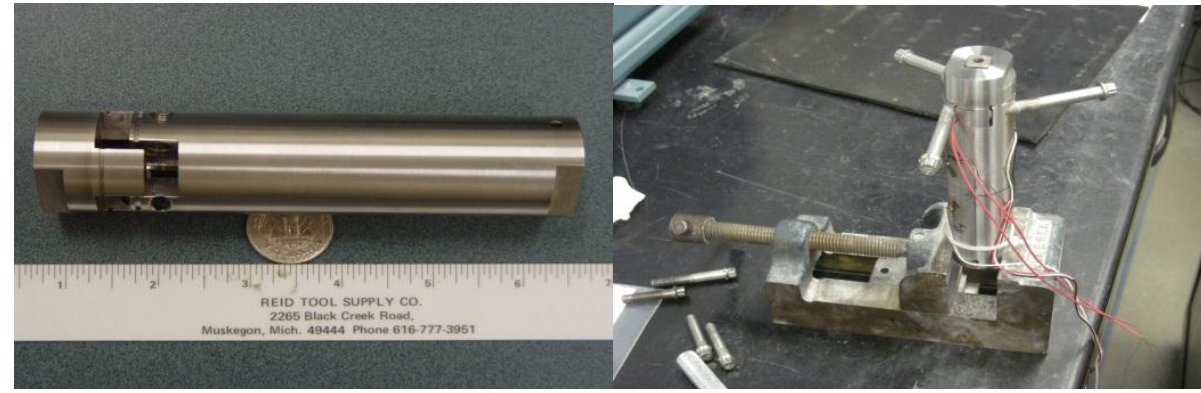

(a) (b)

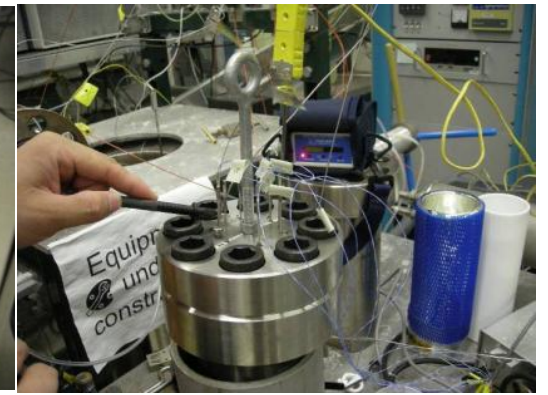

(c)

Figure 11. The SNTT in situ apparatus, (a) in-situ torsion coupler holder, (b) out-of-cell in-situ SNTT device calibration, and (c) a hydrogen pressure tank used in this project. It's noted that the set-up of a full bridge strain gages on the surface of the torsion coupler holder is acting as a torsion load cell.

After being tested in air to establish a torque-strain calibration curve (Figure 12), the SNTT sample was installed in 
the in-situ load frame. The deformation of the sample can be calculated from the strain measured on the load frame and the preload can be obtained from the calibration curve (Figure12). After a sample was preloaded, the load frame was then installed in the hydrogen chamber. After the stabilization of the strain gages, hydrogen was charged to a pre-selected pressure. The sample was held at the preload in hydrogen until fracture occurred, which was determined from the readings of the strain gages.

Figure 13 shows the results from an in-situ SNTT testing. After the strain gages stabilized from the thermal drift, hydrogen was charged to $\sim 2250 \mathrm{psi}$, which resulted in a jump in the strain gage reading due to pressurization. The strain gages stabilized at $313 \mu \varepsilon$ after about $10 \mathrm{hrs}$ in hydrogen and then showed a sudden drop in reading, indicating the

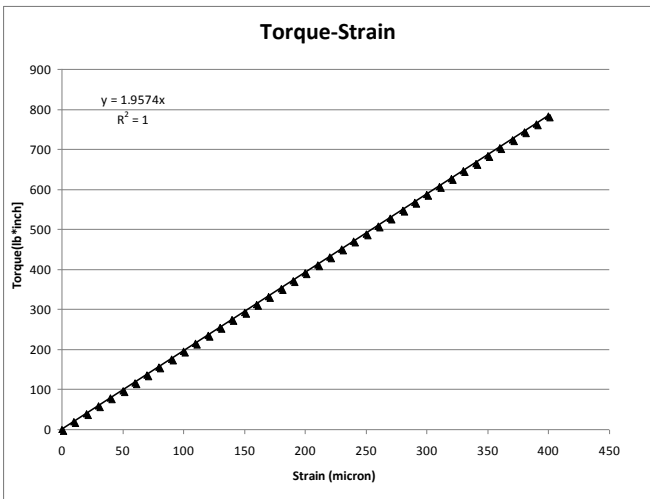

Figure 12. A torque-strain calibration curve of the torsion frame. sample fractured. Hydrogen was vented and samples were removed thereafter. A negative peak was observed in the strain during the venting step similar to that observed during charging (Figure 13).

\subsection{Baseline Specimen Testing in Hydrogen}

Fracture surfaces of notched 4340 SNTT samples failed in the hydrogen chamber showed a typical brittle fracture feature, where a large amount of transgranular fractures was observed (Figure 14). Identical 4340 steel samples were tested both in air and in hydrogen, and the associated micrographs of fractured samples are shown in Figure 14. The sample tested in air exhibited a mixed fracture with both ductile and brittle features. The sample tested in hydrogen showed primarily brittle fracture features near the notch root (where fracture started) and mixed brittle/ductile features in the center region of

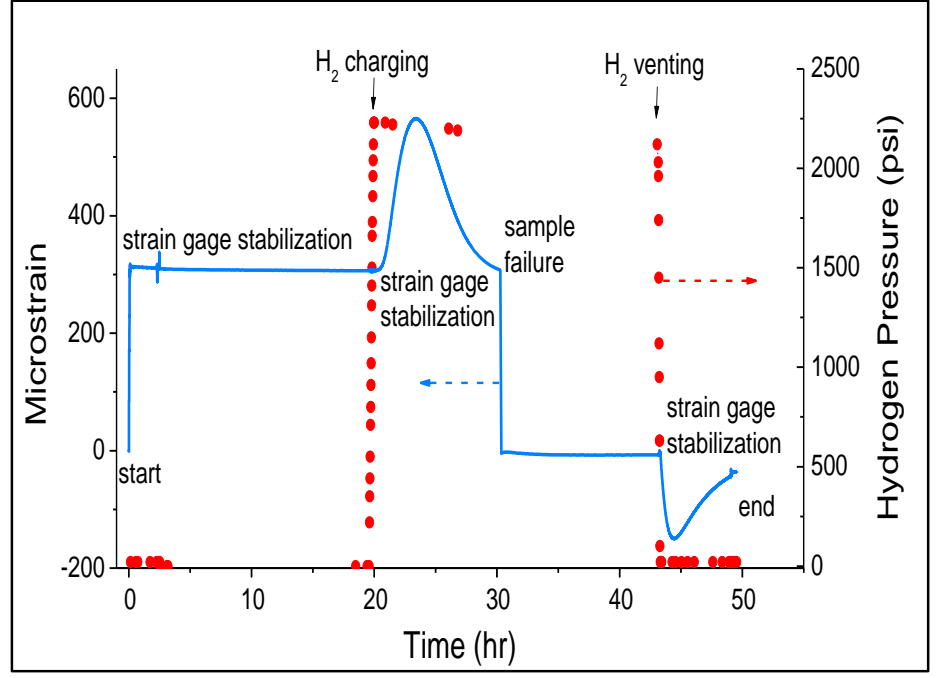

Figure 13. Strain gage readings and hydrogen pressures during a typical SNTT test in the hydrogen chamber. the sample. Hydrogen embrittlement was evident by comparing these two types of tested samples.

A significant drop in the failure load was noticed in the samples test in hydrogen, while the upper limit for the fracture toughness in hydrogen decreased by up to $55 \%$ (Table 2). [23]

\subsection{Testing of Simulated Weld in Hydrogen}

To create simulated weld structures, 4340 samples were treated using a Gleeble machine: (1) heated to $150^{\circ} \mathrm{C}$ in $5 \mathrm{sec}$ and held for $2 \mathrm{sec},(2)$ heated to $950^{\circ} \mathrm{C}$ in $9.75 \mathrm{sec}$ and held for $5 \mathrm{sec},(3)$ helium gas quenched to room temperature. Fracture load in air was
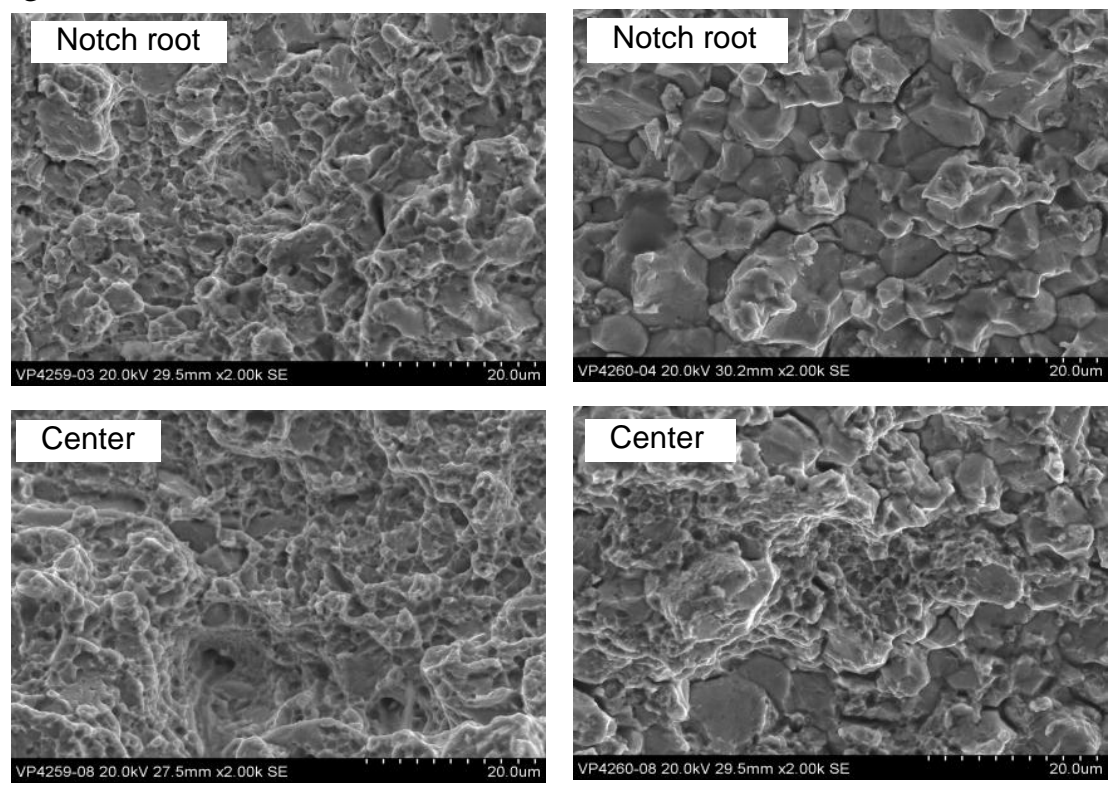

Figure 14. SEM micrographs of fracture surface of 4340 steel samples tested by in situ torsion load frame: (Left) in air and (Right) in hydrogen. 
determined from monotonic loading with a loading rate of $20 \mathrm{lbf}-\mathrm{in} / \mathrm{sec}$. Fracture load in hydrogen was determined from the pre-load. Without cyclic fatigue, the decrease of fracture toughness in hydrogen was as high as $52 \%$ compared to that measured in air, as shown in Table 3.

The SNTT fatigue precrack procedure was also applied to the simulated weld specimens. Samples were cycle fatigued between 15 and $150 \mathrm{lbf}$-in with a frequency of 1 $\mathrm{Hz}$ for 40000 cycles. Fracture toughness values were less than those samples without cycle fatigue, which demonstrated the effect of crack tip sharpening. The decrease in fracture toughness was approximately $75 \%$ in hydrogen compared to that measured in air.

Table 3. Comparison of fracture toughness values of simulated weld SNTT samples tested in air and in hydrogen.

\begin{tabular}{|l|l|c|c|c|c|}
\hline $\begin{array}{c}\text { Sample } \\
\text { ID }\end{array}$ & $\begin{array}{c}\text { Testing } \\
\text { condition }\end{array}$ & Fatigue Precrack & $\begin{array}{c}\text { Fracture torque } \\
(\text { lbf-in) }\end{array}$ & $\begin{array}{c}\text { Fracture toughness } \\
\left(\mathrm{ksi}^{1 / 2}{ }^{1 / 2}\right)\end{array}$ & $\begin{array}{c}\text { Fracture toughness } \\
\text { reduction (\%) }\end{array}$ \\
\hline$\# 1$ & Air & No & 560 & 45.7 & 52 \\
\hline$\# 2$ & $\mathrm{H}_{2}, 1800 \mathrm{psi}$ & No & 260 & 22.0 & \\
\hline$\# 3$ & Air & $\begin{array}{c}15-150 \mathrm{lbf}-\mathrm{in}, 1 \mathrm{~Hz}, \\
40000 \text { cycles }\end{array}$ & 474 & 38.7 & 75 \\
\hline$\# 4$ & H2,1800psi & $\begin{array}{c}15-150 \mathrm{lbf}-\mathrm{in}, 1 \mathrm{~Hz}, \\
40000 \text { cycles }\end{array}$ & 117 & 9.6 & \\
\hline
\end{tabular}

These testing results have successfully demonstrated the applicability of SNTT method in hydrogen environments using this in situ apparatus. However, due to the unstable performance of the strain gages in pressurized hydrogen media, it was difficult to monitor the strain except to detect the final failure. Other strain measurement techniques are currently being investigated.

\section{CONCLUSIONS}

SNTT methodology and its fatigue pre-crack protocol have been successfully applied to structural materials, including metallic, ceramic, and polymeric composite. In order to extend SNTT into hydrogen embrittlement arena. A new in-situ apparatus of utilizing SNTT method was developed for hydrogen embrittlement study. Using the in situ SNTT test, fracture toughness for heat-treated and quenched 4340 high strength steel was found to be reduced at least up to $55 \%$ when exposed to gaseous hydrogen at 2,250 psi. The results of notch tensile testing exposed to hydrogen reveals microstructural evidence of hydrogen embrittlement. SNTT testing in air of samples subject to the simulated welding heat treatment indicates that the microstructure change induced by welding can result in significant reduction of the fracture toughness of pipeline steels. Testing of welded samples in hydrogen is planned in the near future.

\section{ACKNOWLEDGMENTS}

This project is sponsored by U.S. Department of Energy (DOE). Oak Ridge National Laboratory is managed by UT-Battelle, LLC for the U.S. Department of Energy under Contract DE-AC05-00OR22725. The authors also acknowledge Larry Anovitz, Hanbing Xu, Hao Jiang, Thomas Cox, Wei Zhang, and Zhili Feng for their contributions during the program development.

\section{REFERENCE}

1. ASTM Test Method for Plane-Strain Fracture Toughness of Metallic Materials (E399).

2. J. A. Wang, K. C. Liu, D. E. McCabe, and S. A. David, "Using Torsion Bar Testing to Determine Fracture Toughness, KIC,"Fatigue \& Fracture for Engineering Materials and Structure, Vol. 23, pp 45-56, 2000.

3. J. A. Wang, K. C. Liu, and D. E. McCabe, "An Innovative Technique for Measuring Fracture Toughness of Metallic and Ceramic Materials," Fatigue and Fracture Mechanics: $33^{\text {rd }}$ Volume, ASTM STP 1417, W. G. 
Reuter and R. S. Piascik, Eds., pp. 757-770, December 2002.

4. J. A. Wang and K. C. Liu, "A New Approach to Evaluate Fracture Toughness of Structural Materials," Journal of Pressure Vessel Technology, Vol. 126, pp 534-540, November, 2004.

5. Jy-An Wang and Ken Liu, "An Innovative Technique for Evaluating Fracture Toughness of Graphite Materials," Journal of Nuclear Materials Vol. 381 (2008) p.177-184.

6. T. Tan, F. Ren, J-A. Wang, L-C. Edgar, P. Agastra, J. Mandell, W.D. Bertelsen, and C.M. LaFrance, "Investigating fracture behavior of polymer and polymeric composites using the Spiral Notch Torsion Test", Engineering Fracture Mechanics, Vol. 101, p. 109-128, 2013.

7. Parker, E., Brittle Behavior of Engineering Structures, Wiely, New York.

8. Chung, H. S., Takahashi, T., and Suzuki, M., A Microstructural Gradient in HAZ and its Influence Upon the HAZ Fracture Toughness, Welding World, Vol. 16, pp. 248-261, 1978.

9. Koo, J. Y., and Ozekcin, A., A Local Brittle Zone Microstructure and Toughness in Structure Steel Weldments, Proceedings of an International Symposium on Welding Metallurgy of Structural Steels, edited by J. Y. Koo, February 1987, Denver, Colorado.

10. Lundin, C. D., A Fundamental of Weld Discontinuities and Their Significance, Welding Research Bulletin 295 1986.

11. Dolby, R.E., and Archer, G. L., The Assessment of Heat-Affected Zone Fracture Toughness, Proceedings of the Practical Applications of Fracture Mechanics to Pressure Vessel Technology Conference, May 1971.

12. Tronskar, J. P., An Evaluation of Methods to Predict Safe Welding Conditions and Maximum HAZ Hardness in Steel Welding, Journal of Offshore Mechanics and Arctic Engineering, Vol. 117, February 1995, pp. 46-56.

13. R.E. Dolby and G.G. Saunders, "A Review of the Problems of Rehear Cracking in Nuclear Vessel Steels," The Welding Institute, Report No. 18/1876/M, Abington, 1976.

14. A. T. Fikkers and T. Muller, "Hydrogen induced cracking in weld metal," Welding in the World, 143, 1976.

15. Jy-An John Wang, Ian G. Wright, Michael J. Lance, Ken C. Liu, "A New Approach for Evaluating Thin Film Interface Fracture Toughness," Material Science and Engineering A, 426 (2006), 332-345.

16. T. Tan, F. Ren, J-A. Wang, L-C. Edgar, P. Agastra, J. Mandell, W.D. Bertelsen, and C.M. LaFrance, "Investigating fracture behavior of polymer and polymeric composites using the Spiral Notch Torsion Test", Engineering Fracture Mechanics, Vol. 101, p. 109-128, 2013.

17. Jy-An Wang, Fei Ren, Ting Tan, et.al., "An Innovative Technique for Evaluating the Integrity and Durability of Wind Turbine Blade Composites - FY2011 Project Report," ORNL/TM-2011/449, October 2011.

18. H-X. Li, R.H. Jones, J.P. Hirth, D.S. Gelles, Fracture toughness of the F-82H steel-effect of loading modes, hydrogen, and temperature, Journal of Nuclear Materials, 233, (o), pp. 258-263, 1998.

19. US Department of Energy, "National Hydrogen Energy Roadmap," Nov. 2002, available at http://www.eere.energy.gov/hydrogenandfuelcells/pdfs/national_h2_roadmap.pdf

20. US Department of Energy, "Hydrogen, Fuel Cells \& Infrastructure Technologies Program Multi-Year Research, Development and Demonstration Plan," January 21, 2005, page 3-40. Available at http:// www.eere.energy.gov/hydrogenandfuelcells/mypp/

21. U.S. Department of Energy, "Basic Research Needs for the Hydrogen Economy," Basic Energy Sciences Workshop on Hydrogen Production, Storage, and Use, Second Print, February, 2004.

22. Xu, K. "Evaluation of API 5L X80 in High Pressure Hydrogen Gas," ASTM G1.06 Hydrogen Embrittlement Workshop, Nov 8, 2005, Dallas, TX

23. P. S. Lam, R. L. Sindelar, A. J. Duncan, and T. M. Adams, "Literature Survey of Gaseous Hydrogen Effects on the Mechanical Properties of Carbon and Low Alloy Steels,", ASME Journal of Pressure Vessel Technology, 131, pp. 041408-1 to 041408-14, 2009.

24. Jy-An Wang, Ting Tan, Hao Jiang, Wei Zhang, Zhili Feng, "Developing Fatigue Pre-crack Procedure to Evaluate Fracture Toughness of Pipeline Steels Using Spiral Notch Torsion Test," ORNL/TM-2012/337, November 2012.

25. Dynamic Systems Inc., http://www.gleeble.com/.

26. W. Zhang, Z. Feng, and J-A. Wang, "Measurement of fracture toughness of materials with non-uniform microstructure based on spiral notch torsion test", in Proceeding of the ASME 2010 Pressure Vessels and Piping Division/K-PVP conference, (2010) art. no. PVP2010-26112.

27. U.S. Patent 8,176,795 B2. "Apparatuses for Prestressing Rod-Type Specimens in torsion for In-Situ Passive Fracture Toughness Testing in an Extremely High-Pressure Environment of Hydrogen,” May 15, 2012 